\title{
Modeling Homophily Over Time With an Actor-Partner Interdependence Model
}

\author{
Danielle Popp and Brett Laursen \\ Florida Atlantic University
}

\author{
Margaret Kerr, Håkan Stattin, and William J. Burk \\ Örebro University
}

\begin{abstract}
Selection and socialization have been implicated in friendship homophily, but the relative contributions of each are difficult to measure simultaneously because of the nonindependent nature of the data. To address this problem, the authors applied a multiple-groups longitudinal actor-partner interdependence model (D. A. Kashy \& D. A. Kenny, 2000) for distinguishable dyads to 3 consecutive years of intoxication frequency data from a large community-based sample of Swedish youth. Participants, ranging from 12 to 18 years old $(M=14.35, S D=1.56)$ at the start of the study, included 902 adolescents (426 girls and 476 boys) with at least one reciprocated friend during at least one time point and 212 adolescents ( 84 girls and 128 boys) without reciprocated friends at any time. Similarity estimates indicated strong effects for selection and socialization in friends' intoxication frequency. Over time, younger members of these dyads had less stable patterns of intoxication than older members, largely because younger partners changed their drinking behavior to resemble that of older partners.
\end{abstract}

Keywords: homophily, actor-partner interdependence model, friendship formation, nonindependence

Scholars have long recognized that individuals who affiliate with one another tend to be similar on key behaviors, traits, and attributes (Lazarsfeld \& Merton, 1954). The question arising from this observation is tantalizingly simple: What are the origins of this similarity? Of particular interest is similarity between friends because of the widespread concern that some friends may promote antisocial behavior. Concerns about deviant socialization have proven difficult to document, however, because friendship similarity may also be a product of the tendency of youth to select friends who behave in a similar manner (Cairns \& Cairns, 1994). The present study was designed to identify the relative contribution of selection and socialization to friendship homophily. A longitudinal actor-partner interdependence model (APIM; Cook \& Kenny, 2005; Kashy \& Kenny, 2000; Kenny \& Cook, 1999), modified for multiple groups, described similarity and change in friends' intoxication frequency across 3 consecutive years.

Homophily is a dyadic construct that describes similarity between friends. Two distinct processes account for this similarity. Selection emphasizes preexisting similarities that are the basis for the establishment of a friendship. Socialization emphasizes resultant similarities that are the product of participation in a friendship.

Danielle Popp and Brett Laursen, Department of Psychology, Florida Atlantic University; Margaret Kerr, Håkan Stattin, and William J. Burk, Department of Behavioural, Social and Legal Sciences, Örebro University, Örebro, Sweden.

Support for the preparation of this article was provided to Brett Laursen by the National Institute of Mental Health (Grant MH058116). Support for the 10 to 18 Project was provided to Margaret Kerr and Håkan Stattin by the Swedish Research Council and the Bank of Sweden Tercentenary Foundation.

Correspondence concerning this article should be addressed to Danielle Popp, Department of Psychology, Florida Atlantic University, 2912 College Avenue, Davie, FL 33314. E-mail: dpopp1@fau.edu
It seems likely that both contribute to homophily: Youth select friends who are similar on salient characteristics, and they socialize one another in a manner that increases these similarities.

An early study of homophily implicates selection and socialization in the deviant behavior of high school friends (Kandel, 1978). Similarity was measured with Kendall's $\tau$ at two time points across the academic year. Selection effects were represented by similarity estimates before and after the establishment of friendship for those who were not friends at the outset but who became friends later. Socialization effects were represented by similarity estimates at both time points for those who remained friends over the course of the study. Youths who became friends during the spring semester were somewhat more similar on marijuana use and minor delinquency during the fall semester (prior to becoming friends) than those who were friends during the fall semester but not during the spring semester, suggesting selection effects. There was a slight increase in marijuana use and minor delinquency similarity scores among those who remained friends over the course of the school year, suggesting socialization effects. Recent longitudinal studies have focused primarily on socialization effects, employing regression analyses to control or partial out potential selection effects at a prior time point to estimate socialization effects at a subsequent time point. These reports confirm modest but statistically significant socialization effects on several indices of deviance: (a) Stable friends became more similar over time in terms of self-rated (but not teacher-rated) disruption (Berndt \& Keefe, 1995); (b) stable friends were more likely to experiment with cigarettes, drink alcohol, and get drunk regularly if their friends had previously done so (Urberg, Değirmencioğlu, \& Pilgrim, 1997); and (c) stable friends were more likely to become sexually active and to engage in binge drinking if their friends recently reported initiating the same activities (Jaccard, Blanton, \& Dodge, 2005). Similar findings emerge from social network analyses that employ stochastic models to estimate influence and change in drinking behaviors among members of a peer group (Steglich, Snijders, \& West, 2006). 
Taken together, there is evidence of homophily in the deviant behavior of friends, but methodological limitations temper conclusions about the relative importance of selection and socialization. The friendships studied typically involved children in the same grade attending the same school. Given that most antisocial activities take place somewhere other than school, prior research may have underestimated selection effects and socialization effects. Another concern involves the failure to consider whether changes in homophily are a product of normative increases in adolescent antisocial activities (Moffitt, 1993). The one study that examined longitudinal changes at the population level concluded that some but not all of the increase in friend antisocial activities was the product of age-specific increases in delinquency in the sample as a whole (Jaccard et al., 2005). Yet another concern is the failure to identify individuals who are susceptible to peer influence. Previous studies have assumed that friends are mutually influential, but it may be the case that increases in similarity may be traced to change in a particular member of the dyad. Further, previous studies have conflated characteristics of the individual and characteristics of the friendship group. Kandel (1978) analyzed stable and unstable friendships separately. Moreover, other studies have excluded individuals who changed friends or did not have friends, consequently estimating homophily only among stable friendships. This represents a form of sample selection bias such that the individuals who were included were selected on the very behavior being studied (Heckman, 1979). This type of selection bias results in numerous statistical problems, including biased coefficients and a lack of generalizability. Finally, little is known about selection effects because they are usually treated as statistical error to be controlled and the effects of friendship selection statistically partialed out (e.g., Berndt \& Keefe, 1995; Jaccard et al., 2005; Urberg et al., 1997). The only study that attempted to estimate selection (Kandel, 1978) concluded that selection rivals socialization in terms of its contribution to delinquent homophily, but this claim was made on the basis of data that confounded similarity estimates with friendship groups. To date, no study has simultaneously estimated selection and socialization in the same group of friends.

At the heart of the difficulty in disentangling selection effects from socialization effects are the unique methodological issues posed by interdependent data (Laursen, Popp, Burk, Kerr, \& Stattin, in press). Homophily presumes that friends behave in an interdependent manner, meaning that participant reports of behavior are correlated and not statistically independent. Traditional statistical techniques are designed for independent data. Violation of the independence assumption introduces systematic bias into significance tests (Kenny, 1995). The type of error depends on the direction of the association. In the case of homophily, where reports are positively correlated, conventional statistical techniques tend to inflate Type II error in estimates of association. Further, even when researchers suspect that partner influence matters and obtain data from both members of a dyad, often path analyses are performed at the individual level with partner variables as a predictor of individual behavior (i.e., one friend's behavior influences the other, but not vice versa). Such path analyses, in which peer behaviors are used to predict individual behaviors, have a tendency to compound this problem because model misspecification biases parameter estimates and fosters conceptual confusion about influence mechanisms.
The APIM is a data analytic technique designed for nonindependent data, which simultaneously estimates the effect that an individual's predictor variable has on his or her own outcome variable and on his or her partner's outcome variable, partialing out variance shared across participants in the predictor variable. The technique was originally developed to model concurrent effects. In its most common application, the APIM partitions variance shared across reporters and within reporters on the same variable from variance that uniquely describes associations between different reporters on different variables. The technique was recently modified for use with longitudinal data to estimate the stability of mother-child attachment-related views (Cook \& Kenny, 2005) and the stability of aggression among children with and without friends (Adams, Bukowski, \& Bagwell, 2005). This modified procedure requires identical forms of data from both members of the dyad. The longitudinal APIM provides an unbiased estimate of individual stability by separating over-time variance that is unique to individuals from concurrent and over-time variance that is shared by members of the dyad.

In the present study, longitudinal APIM analyses are conducted on three waves of data for friends' reports of intoxication frequency. Figure 1 describes a model for distinguishable dyads in which members of each dyad are distinguished according to their relative age. Effects are modeled simultaneously, partialing out each of the other effects. Time 1 dyadic similarity represents concurrent correlations between members of the dyad on reports of intoxication $(c 1)$. Time 2 residual similarity ( $(2)$ and Time 3 residual similarity (c3) describe concurrent correlations between members of the dyad on the residuals of intoxication frequency. It is important to note that estimates of residual similarity ( $c 2$ and $c 3$ ) are not simply error. Specification of a correlation between residuals controls for additional sources of nonindependence such as unobserved variables. Initial stability of individual behavior is estimated across the first two time points for the older $\left(a l_{o}\right)$ and younger $\left(a l_{y}\right)$ members of the dyad. Subsequent stability of individual behavior is estimated across the second and third time points for the older $\left(a 2_{o}\right)$ and younger $\left(a 2_{y}\right)$ members of the dyad. Initial partner influence describes associations between the older partner's behavior at Time 1 and the younger partner's behavior at Time $2\left(p l_{y}\right)$ and between the younger partner's behavior at Time 1 and the older partner's behavior at Time $2\left(p 1_{o}\right)$. Subsequent partner influence describes associations between the older partner's reports of intoxication frequency at Time 2 and the younger partner's reports at Time $3\left(p 2_{y}\right)$ and between the younger partner's reports of intoxication frequency at Time 2 and the older partner's reports at Time $3\left(p 2{ }_{o}\right)$. The paths are labeled according to traditional APIM nomenclature, where stability estimates represent actor effects $(a)$ and influence estimates represent partner effects $(p)$.

It is important to note that Time 2 residual similarity $(c 2)$ and Time 3 residual similarity $(c 3)$ do not assess changes in dyadic similarity over time. Nor are they strictly comparable to Time 1 estimates of similarity $(c 1)$, because the initial estimate is a correlation between raw scores, whereas the two later estimates are correlations between residual terms. Residual correlations ( $c 2$ and c3) represent any remaining nonindependence in the dyad after partialing out individual stability and partner influence effects including error and unobservable variables. To overcome this limitation, we compute Time 2 and Time 3 similarity estimates by concurrent correlations between raw scores. Of course, concurrent correlations inflate similarity because they include stability and 


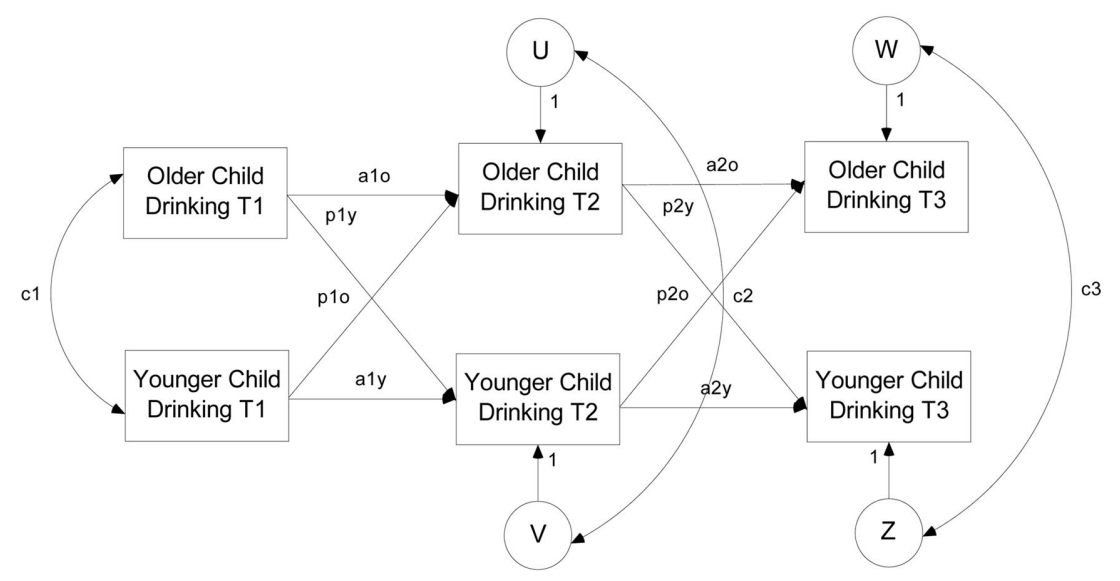

Figure 1. Longitudinal actor-partner interdependence model of alcohol intoxication frequency for dyads distinguished by relative partner age. $a l_{o}=$ initial stability of individual behavior for older child; $a l_{y}=$ initial stability of individual behavior for younger child; $a 2_{o}=$ subsequent stability of individual behavior for older child; $a 2_{y}=$ subsequent stability of individual behavior for younger child; $p 1_{o}=$ initial partner influence for older child; $p 1_{y}=$ initial partner influence for younger child; $p 2_{o}=$ subsequent partner influence for older child; $p 2_{y}=$ subsequent partner influence for younger child. $c 1=$ Time 1 dyadic similarity; $c 2=$ Time 2 residual similarity; $c 3=$ Time 3 residual similarity. $U=$ residual variance in older child's intoxication frequency at Time 2; $V=$ residual variance in younger child's intoxication frequency at Time $2 ; W=$ residual variance in older child's intoxication frequency at Time $3 ; Z=$ residual variance in younger child's intoxication frequency at Time 3 .

partner influence effects, but they do provide a common metric for comparing results. Finally, whenever possible, supplemental analyses that describe the proportions of Time 2 and Time 3 similarity estimates that are due to partner influences and individual stability are performed (adapted from Kenny, Kashy, \& Cook, 2006). These modified APIM correlation proportion scores provide a more precise point for contrasting Time 2 and Time 3 similarity scores across groups by examining similarity due only to partner influence and individual stability.

Recent advances in structural equation modeling (SEM) give rise to a multiple-groups APIM procedure that contrasts specific paths within and between subgroups of distinguishable dyads. This application is well suited for an examination of homophily because it is necessary to examine patterns of similarity in friendships that begin and end at different time points in order to disentangle selection effects from socialization effects. Selection effects are operationalized in terms of concurrent associations between two individuals during the time preceding the establishment of the friendship. To isolate selection effects, one must estimate similarity prior to the start of the friendship, controlling for subsequent socialization effects. Socialization effects are operationalized in terms of concurrent associations between two individuals during the time following the establishment of the friendship. To isolate socialization effects, one must estimate similarity after the start of the friendship, controlling for prior selection effects. When three data points are available, selection effects and socialization effects can be simultaneously estimated for three groups: (a) dyads who were friends at Time 2 and at Time 3 but not at Time 1; (b) dyads who were friends at Time 2 but not at Time 1 or at Time 3 ; and (c) dyads who were friends at Time 3 but not at Time 1 and at Time 2. The present study also contains estimates of homophily for three comparison groups of randomly paired same-grade, same-gender youth, none of whom had ever nominated the other as a friend or peer associate during the course of the study. Analyses including these groups will provide a benchmark against which selection effects and socialization effects can be compared. Rates of alcohol consumption increase across the adolescent years, so it is important to estimate similarity between randomly paired youth to rule out the possibility that increasing similarity between friends is the result of age-related increases in similarity within the population. Previous studies (Steglich et al., 2006) of peer networks suggest that both selection and socialization play a role in alcohol use, and we expected similar findings in our study of friends.

The present study utilizes a distinguishable dyad framework, wherein each member of a dyad belongs to a unique class of participants. Cross-lagged associations denote whether partners are similarly influential or whether one class of participants is more influential than the other. To demonstrate, we dichotomized partners according to age, categorizing one member as older and the other as younger. The tendency for older partners to influence younger partners is represented by associations between the older partner's antecedent behavior and the younger partner's subsequent behavior $\left(p 1_{y}\right.$ and $\left.p 2_{y}\right)$, whereas the tendency for younger partners to influence older partners is represented by associations between the younger partner's antecedent behavior and the older partner's subsequent behavior $\left(p 1_{o}\right.$ and $\left.p 2_{o}\right)$. Stability estimates should be related to influence estimates in that individuals who are more susceptible to influence will demonstrate less stable drinking patterns. We expected that intoxication frequency among younger members of the dyad would be less stable than that among older members of the dyad because we anticipated that younger adolescents would be influenced by the prior behavior of older adolescents but not the reverse.

It is important to note the assumptions of our model. The analyses were conducted using SEM with AMOS (Arbuckle, 2006). Standard SEM assumptions apply, including those concern- 
ing multivariate normality, minimum sample size requirements, and identification procedures (see Kline, 2005). Multiple model procedures do not permit the same individual to be represented more than once in the data set, so the analyses limited participants to a single friendship, regardless of their actual number of friends.

\section{Method}

\section{Participants}

The sample included 1,090 adolescents (546 girls and 544 boys) drawn from a community-based sample of youth attending four schools in a small city (population 26,000) in central Sweden. The present sample included students from 38 classrooms who, at the outset of this study, were in either the 7th grade (range: 12 to 15 years old, $M=13.07, S D=0.20$ ) or the 10 th grade (range $=15$ to 18 years old, $M=16.17, S D=0.41$ ). At the outset of this study, $80.1 \%$ of the adolescents lived with both biological parents, $13.4 \%$ lived with one biological parent and one stepparent (or significant other), and 6.6\% lived in single-parent households. The $6 \%$ unemployment rate in the community at the outset of the study was similar to that in Sweden as a whole; mean incomes were about $4 \%$ lower than those in the rest of the country. Most participants were born in Sweden (93.1\%) and had at least one biological parent who was born in Scandinavia (93.3\%). Approximately $86 \%$ of mothers were employed (57\% full time) and $94 \%$ of fathers were employed ( $91 \%$ full time).

\section{Instruments}

Questionnaires were completed in class during regular school hours. Surveys were administered by trained research assistants. Teachers were not present. This investigation concerns data from surveys that describe peer affiliation and delinquent activities (for details, see Kiesner, Kerr, \& Stattin, 2004; Persson, Kerr, \& Stattin, 2007). Identical questionnaires were completed at each wave of data collection.

Peer nominations. Every year participants identified up to three important peers, who were defined as "someone you talk with, hang out with, and do things with." Participants were instructed that important peers could include friends, siblings, or romantic partners but not parents or other adults. Instructions further indicated that important peers could live in different communities and need not be the same age or the same gender. Participants also indicated whether those nominated were friends, siblings, romantic partners, or others. Reports concerning siblings, romantic partners, and others were not included in the present study. Mutual friends were defined as dyads with reciprocal, concurrent important-peer friend nominations.

In addition to these three important peers, participants also identified up to 20 other peer associations: up to 10 whom they spent time with in school and up to 10 whom they spent time with out of school. These peers could be older or younger, boys or girls, but they could not be adults.

Frequency of intoxication. Each year participants completed three items measuring frequency of intoxication. One item referred to intoxication in the previous year, and two items referred to intoxication with important peers and other peers during the past month (e.g., "Have you ever drunk so much beer, liquor, or wine that you got drunk?"). Responses were scored on a 3-point scale:
$1=$ no, it has never happened $; 2=1$ time $; 3=$ several times Scores were averaged across items. Internal consistency was adequate at each time point ( $\alpha=.85$ to .88$)$.

\section{Procedure}

Students were recruited in classrooms during school hours. They were told what kinds of questions they would be asked and how long it would take to participate. Students were informed that participation was voluntary and confidential; they were assured that their answers would not be revealed to parents, teachers, the police, or anyone else. Parents were informed about the study in community meetings and through the mail, where they received a postage-paid card to return if they did not want their child to participate in the study. Parents and youth were informed that either was free to end participation in the study at any time. Youth were not paid for participation, but all students (participants and nonparticipants) were eligible for class parties and drawings provided by the project.

All students in the community enrolled in Grades 4 to 12 were invited to participate in the study each year. Five annual waves of data were available. Participation in three consecutive waves of data collection was a precondition for inclusion in this investigation, so Time 1 represents data collected during Wave 1, Wave 2, or Wave 3 of the study. Students in Sweden change schools in the 7 th grade and in the 10th grade. To avoid confounding friendship stability groups with school changes, we limited the present investigation to longitudinal data that encompass the 7 th to 9 th grades and the 10th to 12 th grades. A total of 2,370 students were enrolled in Grades 7 and 10 during Waves 1, 2, and 3. Data from 3 consecutive years were available for $63.9 \%(n=1,514)$ of these students. A total of 70 students were excluded because of incomplete friendship nomination data. Logistic regression analyses examined whether age, ethnicity, gender, family structure, or intoxication frequency predicted attrition. Significant results emerged for adolescent age (odds ratio $[\mathrm{OR}]=0.78, p<.001$ ), household structure $(\mathrm{OR}=2.74, p<.001)$, and intoxication frequency $(\mathrm{OR}=0.79, p=.006)$. Thus, the final sample had more young adolescents from intact families, who were less frequently intoxicated than those adolescents who were lost to attrition.

The number of participants with missing data was small $(n=$ $61 ; 5.6 \%$ ). Listwise deletion, however, would produce a loss of 122 dyads. Chi-square analyses suggest that missing data were randomly distributed among the friendship groups, $\chi^{2}(5, N=$ $878)=0.997, p=.96$. Therefore missing values at the item level were imputed using the expectation maximization algorithm for participants with incomplete intoxication surveys. Imputation was based on all measures used in this investigation (age, gender, ethnicity, family structure, and intoxication frequency), as well as additional items describing prosocial and antisocial activities, depression, impulsivity, risk-taking behaviors, sexual activity, school adjustment, and self-worth. The algorithm was limited to linear associations between variables. Single imputation was selected instead of multiple imputations to simplify the data analyses. Single imputation comes at the potential cost of underestimating standard errors, which results in greater Type I errors. Single imputation also introduces biases that are not accounted for by traditional methods or statistical software. However, single imputation is useful in cases such as this when the amount of missing 
data is small (McKnight, McKnight, Sidani, \& Figueredo, 2007). Comparisons of adolescents with imputed data against those without imputed data on demographic variables revealed no significant differences on gender, age group, or family structure. Finally, comparisons of adolescents with imputed data against those without imputed data on intoxication frequency at each time point revealed no significant differences.

A total of 1,230 participants reported at least one reciprocated friendship with another member of the study during at least one of the three time periods. Some participants were involved in more than one friendship at a time, and some had different friends at different times. The inclusion of all friendship dyads in the analyses would have violated the independence assumption of multiple-groups modeling and introduced the potential for bias due to unequal contributions by individuals. To avoid these problems, analyses focused on the highest ranked reciprocal friends, with each participant restricted to one and only one friendship dyad. As a consequence, $22.7 \%(n=328)$ of participants with friends were not included in friendship dyads because their reciprocated friends were paired with other partners. This left a final sample of 902 friended adolescents. A chi-square analysis identified grade-level differences between friended participants omitted from friendship groups and friended participants included in friendship groups, $\chi^{2}(1)=11.60, p<.001$. More friended students were omitted from friendship groups in Grade 10 than in Grade 7. There were no statistically significant gender, ethnicity, or family structure differences between friended participants omitted from friendship groups and friended participants included in friendship groups. Independent-sample $t$ tests failed to reveal any statistically significant differences between these groups on intoxication frequency.

Friends were classified into one of seven groups. Three groups of dyads were friends across more than one consecutive time period: (a) Enduring friends ( $n=119$ dyads) were friends at Times 1, 2, and 3; (b) waning friends ( $n=55$ dyads) were friends at Times 1 and 2 but not at Time 3; and (c) nascent friends $(n=$ 57 dyads) were friends at Times 2 and 3 but not at Time 1. Four groups of dyads were friends at one time point only or at nonconsecutive time points: (a) Time 1 friends ( $n=70$ dyads); (b) Time 2 friends ( $n=62$ dyads); (c) Time 3 friends ( $n=76$ dyads); and (d) intermittent friends ( $n=12$ dyads), who were friends at Times 1 and 3 but not at Time 2 . In each case, neither partner nominated the other as a friend at any time point except when the dyad was classified as a reciprocal friendship.

A chi-square analysis identified gender differences in friendship groups, $\chi^{2}(6)=31.11, p<.01$. Follow-up one-way chi-squares adjusted for multiple comparisons indicated that nascent friends and enduring friends contained more female dyads than male dyads and that Time 1 friends and waning friends contained more male dyads than female dyads. There were no statistically significant grade-level differences in friendship groups. Dyadic heterogeneity precluded contrasts of ethnicity and family structure. Power considerations did not permit multiple-groups modeling of friendship groups by gender. Correlational contrasts were conducted, comparing results for boys and girls on each path within the six friendship and three comparison groups, across all three time points. The number of differences did not exceed chance levels ( 6 of 135 possible contrasts were statistically significant at or above the .05 level).
Three comparison groups were created to gauge baseline levels of similarity. The first group included only participants who were not involved in a reciprocated friendship at any time point. This friendless group ( $n=106$ dyads) consisted of random pairs of participants without reciprocated friends. The second group included only participants with a reciprocated friend at one or more of the three time points. This friended group ( $n=439$ dyads) consisted of random pairs of participants with reciprocated friends. The third group included all participants with three consecutive waves of friendship data. This total sample group ( $n=545$ dyads) consisted of random pairs of participants, regardless of friendship status. Comparison group dyads consisted of same-grade, samegender youth; neither partner nominated the other as an important peer or as a peer associate at any time point.

\section{Plan of Analysis}

Preliminary analyses of variance (ANOVAs) examined differences between friendship groups in mean levels of intoxication frequency. Multiple-group SEMs describe stability in individual levels of intoxication frequency, identify partner influences on subsequent intoxication frequency, and delineate selection and socialization effects by specifying concurrent similarity in different friendship groups. Small sample sizes precluded the inclusion of the intermittent friends group in APIM analyses.

Selection effects are evident at Time 1 for nascent friends and for Time 2 friends; selection effects are also evident at Time 2 for Time 3 friends. Because the APIM provides residual correlations for Time 2 and Time 3 dyadic similarity, we also calculated concurrent interclass correlations between partners' reports of intoxication frequency, so that selection effects could be compared across groups and with socialization effects. It should be noted that simple correlations are inflated by individual stability, partner influence, and error; this is a problem that cannot be addressed in Time 1 APIM estimates of similarity or in Time 2 or Time 3 interclass correlations.

Socialization effects are evident at Time 2 for Time 2 friends, at Time 3 for Time 3 friends, and at Times 2 and 3 for nascent friends. Here, too, concurrent interclass correlations were calculated between partners' reports of intoxication frequency, so that socialization effects could be compared across groups and with selection effects. To determine the extent to which similarity among dyad members is due to error or unobservable variables, we calculated a modified APIM correlation proportion score that represents the part of the Time 2 and Time 3 concurrent interclass correlations accounted for by dyadic influence effects and individual stability. ${ }^{1}$ These scores describe the

\footnotetext{
${ }^{1}$ Interclass correlations include variance due to individual stability, partner influence, systematic similarities, and error. Socialization effects are operationalized as the unique effect of partner influences, exclusive of these other forms of variance. To compare socialization effects across groups, we calculated the proportion of the interclass correlation at Time 2 and Time 3 that is explained by partner effects, controlling for actor effects: $a 2_{o} p 2_{y}+a 2_{y} p 2_{o}+r\left(a 2_{o} a 2_{y}+p 2_{o} p 2_{y}\right)$ (adapted from Kenny et al., 2006 p. 146).
} 
part of the socialization effects estimates attributable to dyadic influences and individual stability.

\section{Results}

\section{Preliminary Analyses}

A 6 (friendship group) $\times 2$ (age group: younger vs. older) $\times 3$ (time) repeated measures ANOVA was conducted with intoxication frequency as the dependent variable. A main effect for time, $F(2,865)=142.23, p<.001$, was qualified by an interaction between time and age group, $F(2,865)=16.64, p<.001$. Follow-up $t$ tests indicated that older adolescents reported greater levels of intoxication frequency than younger adolescents at Time $1(M=1.61, S D=0.62$, and $M=1.44, S D=0.67$, respectively $)$ and at Time $2(M=1.69, S D=0.67$, and $M=1.59, S D=0.65$, respectively) but not at Time $3(M=1.83, S D=0.76$, and $M=$ $1.87, S D=0.77$, respectively). Table 1 provides a breakdown of intoxication frequency scores by age and friendship groups.

\section{Longitudinal APIM for Multiple Friendship Groups}

Figure 1 depicts a model with equality constraints across groups on individual stability paths $\left(a 1_{o}, a 1_{y}, a 2_{o}\right.$, and $\left.a 2_{y}\right)$, partner influence paths $\left(p 1_{o}, p 1_{y}, p 2_{o}\right.$, and $\left.p 2_{y}\right)$, the initial concurrent similarity path $(c 1)$, means and variances on initial intoxication, intercepts at Time 2 and Time 3, and residuals. A statistically significant chi-square difference test indicates that patterns of association differ between friendship groups and that the sample should not be collapsed into a single model. The unconstrained model fit the data, $\chi^{2}(24)=70.3, p<.01$ (comparative fit index $[\mathrm{CFI}]=.973$; root-mean-square error of approximation [RMSEA] $=.067$; RMSEA confidence interval $[\mathrm{CI}]=.049-.085$; pclose $=$ .060). Chi-square difference tests revealed statistically significant differences between Time 2 and 3 residual similarity paths, $\chi^{2}(65)=113.4, p<.001$. As a consequence, unconstrained analyses were conducted in which all of the parameter estimates varied freely between the six friendship groups.

Separate models were estimated for each of the three comparison groups. All three models fit the data well: friended dyads, $\chi^{2}(4)=37.9, p<.01(\mathrm{CFI}=.974 ;$ RMSEA $=.139 ;$ pclose $=$ $.000)$; friendless dyads, $\chi^{2}(4)=15.1, p<.01(\mathrm{CFI}=.975$; RMSEA $=.162$; pclose $=.016)$; and total sample, $\chi^{2}(4)=31.3$, $p<.01(\mathrm{CFI}=.984 ;$ RMSEA $=.112$; pclose $=.002)$.

Figures 2 through 10 depict APIM results for all friendship and comparison groups. Table 2 describes interclass correlations for partners' scores of intoxication frequency at each time point. The bulk of our presentation focuses on the three friendship groups that permit simultaneous estimation of selection effects and socialization effects.

Selection effects. Statistically significant selection effects emerged for Time 1 dyadic similarity for nascent friends, Time 1 dyadic similarity for Time 2 friends, and Time 2 residual similarity for Time 3 friends. Interclass correlations were moderate to large across groups ( $r \mathrm{~s}=.53$ to .68 ). The modified APIM proportion score suggested that error and similarity due to unobserved variables accounted for $57 \%$ of the selection effects in the Time 3 friend group (the only group in which this statistic could be calculated). Results for comparison groups indicate that Time 1 dyadic similarity coefficients were statistically significant for the friended, friendless, and total sample groups, but the magnitude of association was small ( $r \mathrm{~s}=.15, .25$, and .16 , respectively). Taken together, the results indicate that a substantial amount of the variance in intoxication frequency is accounted for by similarities that exist prior to the friendship. Population level similarities account for a small fraction of this total.

Socialization effects. Statistically significant socialization effects emerged for Time 2 residual similarity for Time 2 friends, for Time 3 residual similarity for Time 3 friends, and for Time 2 and Time 3 residual similarity for nascent friends. Interclass correlations were moderate to large across groups $(r s=.46$ to .75$)$. The

Table 1

Alcohol Intoxication Frequency by Friendship Group

\begin{tabular}{|c|c|c|c|c|c|c|c|c|c|c|}
\hline \multirow[b]{2}{*}{ Friendship group } & \multicolumn{3}{|c|}{ Time 1} & \multicolumn{3}{|c|}{ Time 2} & \multicolumn{3}{|c|}{ Time 3} & \multirow{2}{*}{$\begin{array}{c}n \text { of } \\
\text { dyads }\end{array}$} \\
\hline & Older & Younger & Total & Older & Younger & Total & Older & Younger & Total & \\
\hline \multicolumn{11}{|l|}{ Stable friends } \\
\hline \multicolumn{11}{|l|}{ Enduring (Time 1, 2, } \\
\hline Nascent (Time $2 \& 3$ ) & $1.59(0.57)$ & $1.59(0.72)$ & $1.59(0.65)$ & $1.70(0.63)$ & $1.71(0.72)$ & $1.70(0.68)$ & $1.96(0.81)$ & $2.13(0.83)$ & $2.05(0.82)$ & 57 \\
\hline Waning (Time $1 \&$ 2) & $1.61(0.62)$ & $1.28(0.56)$ & $1.44(0.61)$ & $1.67(0.70)$ & $1.52(0.61)$ & $1.60(0.66)$ & $1.77(0.77)$ & $1.82(0.76)$ & $1.79(0.76)$ & 55 \\
\hline \multicolumn{11}{|l|}{ Unstable friends } \\
\hline Time 1 only & $1.60(0.56)$ & $1.35(0.62)$ & $1.47(0.60)$ & $1.77(0.67)$ & $1.67(0.72)$ & $1.72(0.69)$ & $1.86(0.72)$ & $1.98(0.79)$ & $1.92(0.76)$ & 70 \\
\hline Time 2 only & $1.46(0.58)$ & $1.39(0.68)$ & $1.42(0.63)$ & $1.59(0.64)$ & $1.53(0.69)$ & $1.56(0.66)$ & $1.67(0.72)$ & $1.87(0.83)$ & $1.77(0.78)$ & 62 \\
\hline Time 3 only & $1.60(0.58)$ & $1.36(0.58)$ & $1.48(0.59)$ & $1.68(0.62)$ & $1.50(0.67)$ & $1.59(0.66)$ & $1.87(0.75)$ & $1.76(0.73)$ & $1.81(0.74)$ & 76 \\
\hline Total & $1.61(0.62)$ & $1.44(0.67)$ & $1.53(0.65)$ & $1.69(0.67)$ & $1.59(0.65)$ & $1.64(0.68)$ & $1.83(0.76)$ & $1.87(0.77)$ & $1.85(0.76)$ & \\
\hline \multicolumn{11}{|l|}{ Comparison } \\
\hline Friended & $1.68(0.62)$ & $1.70(0.62)$ & & $1.68(0.68)$ & $1.45(0.63)$ & & $1.84(0.77)$ & $1.68(0.72)$ & & 439 \\
\hline Friendless & $1.78(0.69)$ & $1.64(0.75)$ & & $1.80(0.70)$ & $1.72(0.71)$ & & $1.93(0.76)$ & $1.83(0.73)$ & & 106 \\
\hline \multicolumn{11}{|l|}{ Friended and } \\
\hline
\end{tabular}

Note. Alcohol intoxication scores range from 1.0 (never) to 3.0 (several times). Values in parentheses are standard deviations. Comparison groups include random pairs of same-grade, same-gender participants, neither of whom had ever nominated the other as an important peer or peer associate. 


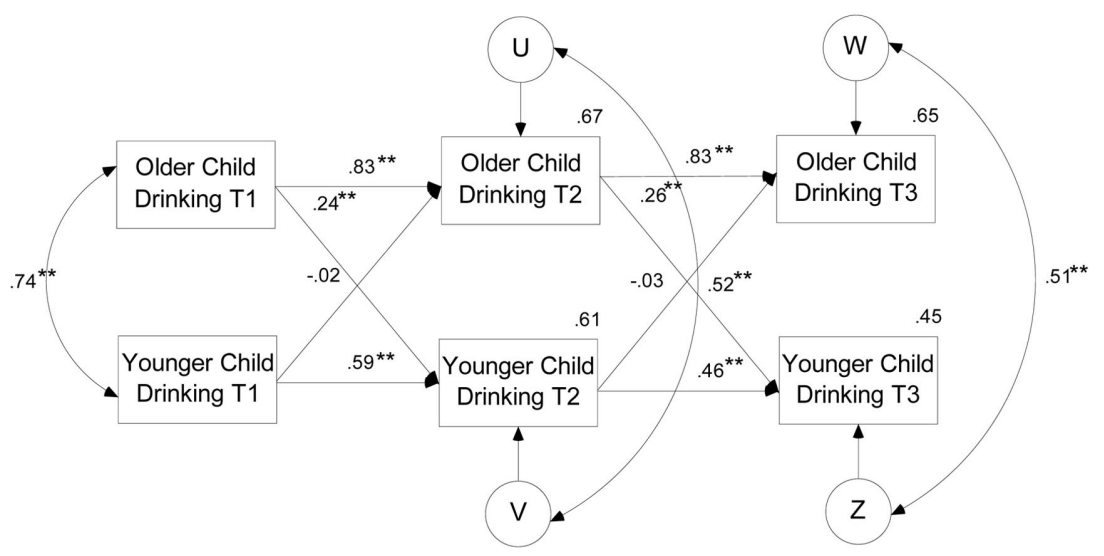

Figure 2. Enduring friendship group model (standardized estimates). $U=$ residual variance in older child's intoxication frequency at Time 2; $V=$ residual variance in younger child's intoxication frequency at Time 2; $W=$ residual variance in older child's intoxication frequency at Time $3 ; Z=$ residual variance in younger child's intoxication frequency at Time $3 .{ }^{*} p<.05 .{ }^{* *} p<.01$.

modified APIM proportion score suggested that dyadic influences and stability account for $51 \%-56 \%$ of the socialization effects in the nascent friend group, $47 \%$ of the socialization effects in the Time 2 friend group, and $32 \%$ of the socialization effects in the Time 3 friend group. Results for comparison groups indicate that dyadic similarity coefficients were statistically significant at Time 2 and Time 3 only for the friendless and total sample groups, but the magnitude of association was small ( $r \mathrm{~s}=.09$ to .21 ). Taken together, the findings indicate that a substantial amount of intoxication frequency homophily is accounted for by similarities that arise after the friendship has been established and that only a small proportion of this variance may be attributed to population level homophily.

Partner influence effects. Statistically significant partner influence effects emerged for the nascent group across the period when the friendship was established $(p 1)$. Older partners' drinking at Time 1, prior to the establishment of the friendship, predicted younger partners' drinking at Time 2 , after the establishment of the friendship. Younger partners had no influence on older partners during this period. This suggests that during the initial phases of friendship formation, older youths influence the drinking behavior of younger youths, but not the reverse. Statistically significant partner effects also emerged for the nascent friend group during the period subsequent to the establishment of the friendship $(p 2)$. Older partners' drinking at Time 2 predicted younger partners' drinking at Time 3, and younger partners' drinking at Time 2 predicted older partners' drinking at Time 3. This suggests that after a friendship is established, older and younger youths mutually influence each other's drinking behavior. Enduring friends also reported partner influence effects after the establishment of the friendship; older friends influenced younger friends, but not the reverse. No partner influence effects emerged for Time 2 friends. For Time 3 friends, partner influence effects emerged prior to the formation of the friendship but not during the course of friendship formation. By way of contrast, none of the partner influence effects were statistically significant for any of the comparison

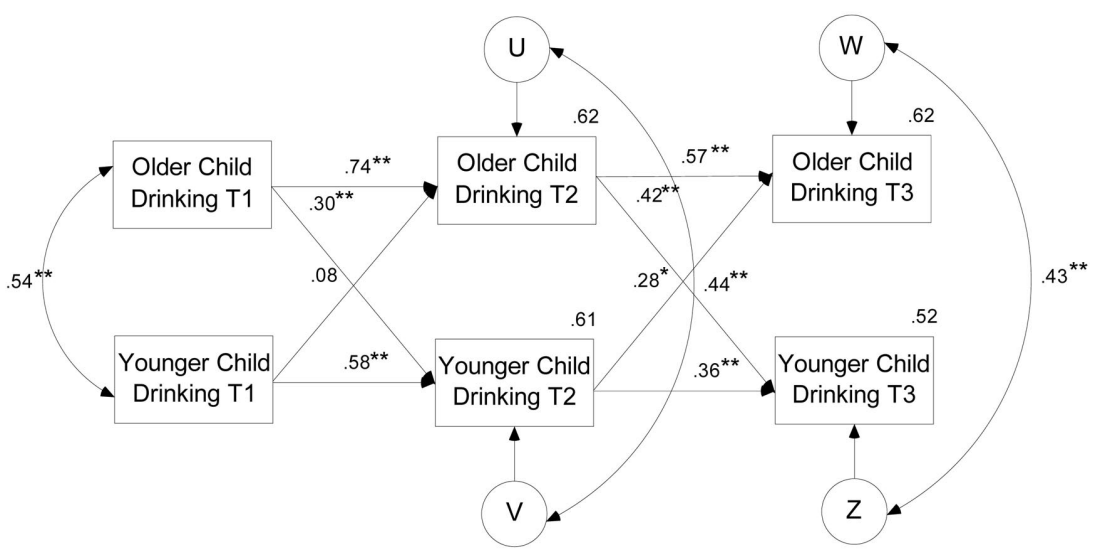

Figure 3. Nascent friendship group model (standardized estimates). $U=$ residual variance in older child's intoxication frequency at Time 2; $V=$ residual variance in younger child's intoxication frequency at Time 2; $W=$ residual variance in older child's intoxication frequency at Time $3 ; Z=$ residual variance in younger child's intoxication frequency at Time $3 .{ }^{*} p<.05 .{ }^{* * *} p<.01$. 


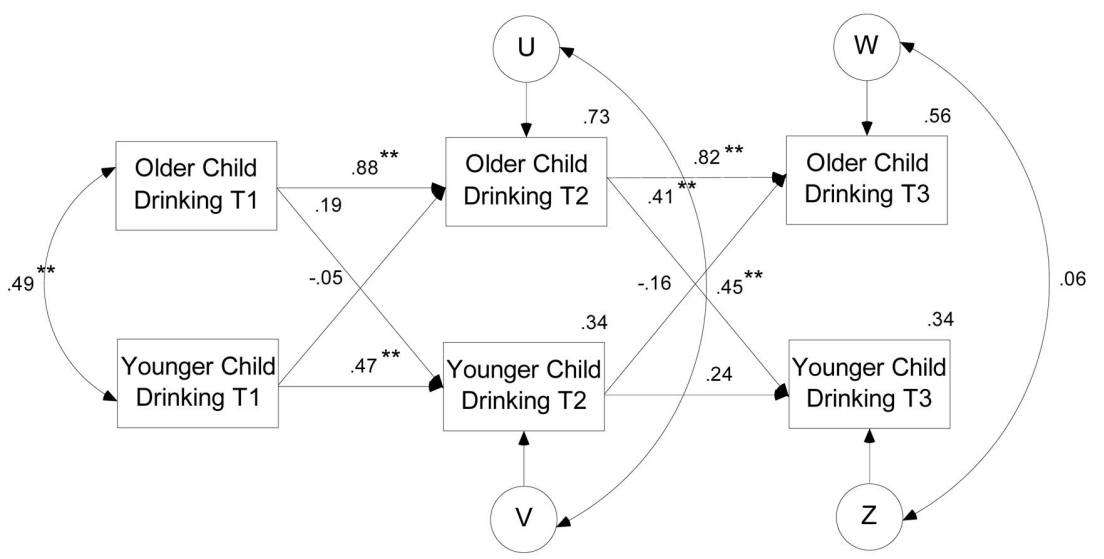

Figure 4. Waning friendship group model (standardized estimates). $U=$ residual variance in older child's intoxication frequency at Time $2 ; V=$ residual variance in younger child's intoxication frequency at Time 2; $W=$ residual variance in older child's intoxication frequency at Time $3 ; Z=$ residual variance in younger child's intoxication frequency at Time $3 .{ }^{*} p<.05 .{ }^{* * *} p<.01$.

groups. Together, these findings indicate that to the extent that older youths are influencing the drinking behavior of younger youths, they are doing so in the context of a friendship; normative developmental trends in alcohol experimentation are not responsible for age-related partner influences.

Individual stability effects. Statistically significant individual stability effects emerged for younger and older partners in all friendship groups. With one exception, older partners always reported more over-time stability in intoxication frequency than younger partners. Similarly, all individual stability effects were significant for the three comparison groups. Further, older partners always reported more over-time stability.

\section{Discussion}

Friendship homophily poses a difficult analytic challenge because the interdependent nature of the data makes it difficult to parse selection effects from socialization effects. Taking advantage of recent advances in multiple-groups SEM, we modified longitudinal APIM procedures for distinguishable dyads to encompass three waves of longitudinal data on intoxication frequency among adolescent friends. As expected, we found that friendships developed among those who reported similar levels of intoxication prior to becoming friends and that friends became more similar in their frequency of intoxication over the course of the friendship. The findings are unique in that they provide the first measure of the relative role played by friendship selection and socialization in the development of problem drinking behavior. Among groups in which both could be estimated, selection effects rivaled socialization effects in terms of their magnitude, validating a claim that Kandel (1978) initially advanced. We also found evidence to suggest that older youths shape the drinking behavior of younger youths during the early stages of friendship formation and beyond;

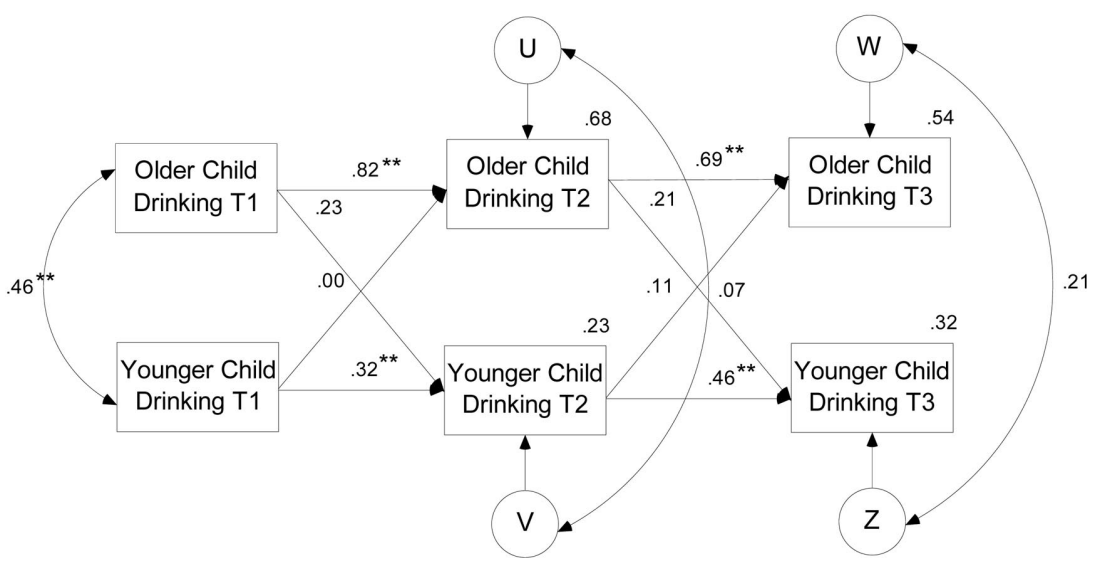

Figure 5. Time 1 only friendship group model (standardized estimates). $U=$ residual variance in older child's intoxication frequency at Time 2; $V=$ residual variance in younger child's intoxication frequency at Time 2; $W=$ residual variance in older child's intoxication frequency at Time $3 ; Z=$ residual variance in younger child's intoxication frequency at Time $3 .{ }^{*} p<.05 .{ }^{* *} p<.01$. 


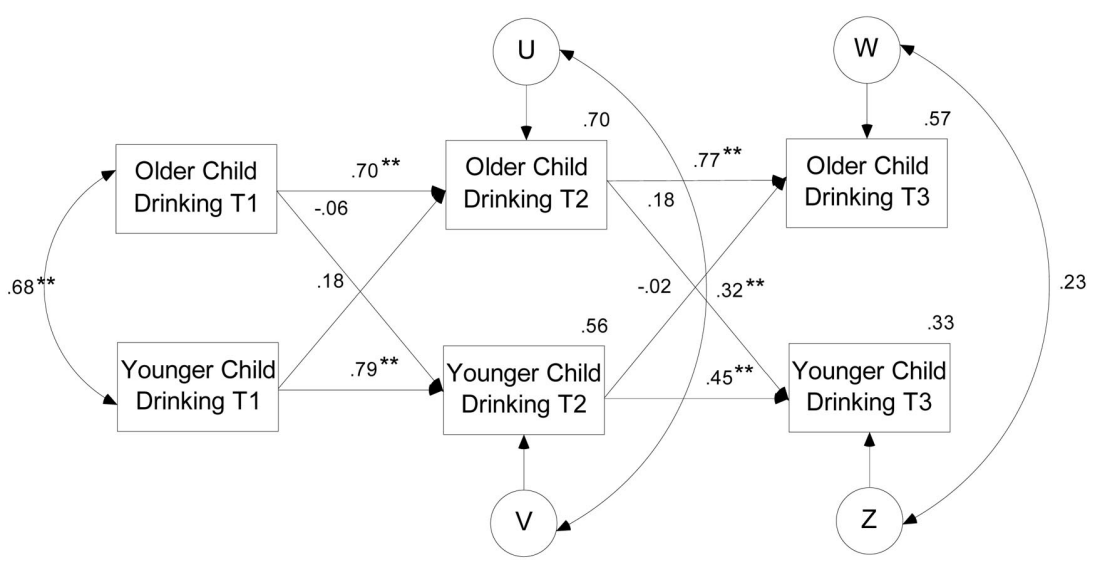

Figure 6. Time 2 only friendship group model (standardized estimates). $U=$ residual variance in older child's intoxication frequency at Time 2; $V=$ residual variance in younger child's intoxication frequency at Time 2; $W=$ residual variance in older child's intoxication frequency at Time $3 ; Z=$ residual variance in younger child's intoxication frequency at Time $3 .{ }^{*} p<.05 .{ }^{* *} p<.01$.

younger youths did not influence the drinking behavior of older youths during the early stages of a friendship, but their influence increased during the later stages of the friendship.

The application of APIM techniques in developmental psychology is a relatively recent phenomenon (Laursen, 2005). Originally designed for use with concurrent data collected from distinguishable dyads, the technique has attracted considerable attention as it has been adapted for use in different developmental contexts (Card, Little, \& Selig, in press). The modified APIM technique described in the present investigation builds on previous studies in which the procedure was adapted for use with friends (Cillessen, Jian, West, \& Laszkowski, 2005), two waves of longitudinal data (Cook \& Kenny, 2005), and multiple groups (Neyer, 2002). Our contribution to this evolving process was to synthesize these advances into a single analytic procedure that addressed friendship homophily. First, we extended the model to include three consecutive time points. The addition of multiple time points and multiple groups made it possible for us to assess similarity at different stages of the friendship process. Second, we estimated selection and similarity effects among dyads in which participants were distinguished on the basis of their relative age. The multiplegroups longitudinal APIM is not limited to distinguishable dyads, however; the procedure can also be applied to indistinguishable dyads by adding equality constraints on the means and variances for the predictor variables, actor paths, partner paths, intercepts, and means and variance of the disturbances (Olsen \& Kenny, 2006). Elsewhere, Laursen and colleagues (in press) describe APIM procedures for estimating homophily in the delinquent behavior of indistinguishable dyads.

This study is also significant because it provides a means for identifying the relative influence of partners. A persistent problem in studies of homophily has been the assumption that friends are similarly influential. The use of APIM techniques for distinguishable dyads is an important step toward identifying the direction of

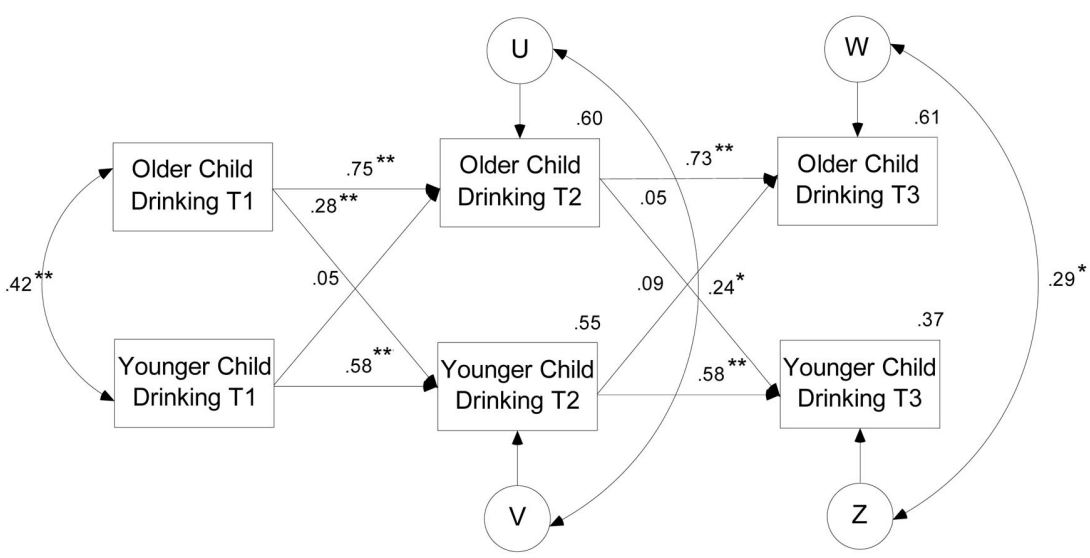

Figure 7. Time 3 only friendship group model (standardized estimates). $U=$ residual variance in older child's intoxication frequency at Time $2 ; V=$ residual variance in younger child's intoxication frequency at Time 2; $W=$ residual variance in older child's intoxication frequency at Time $3 ; Z=$ residual variance in younger child's intoxication frequency at Time $3 .{ }^{*} p<.05 .{ }^{* * *} p<.01$. 


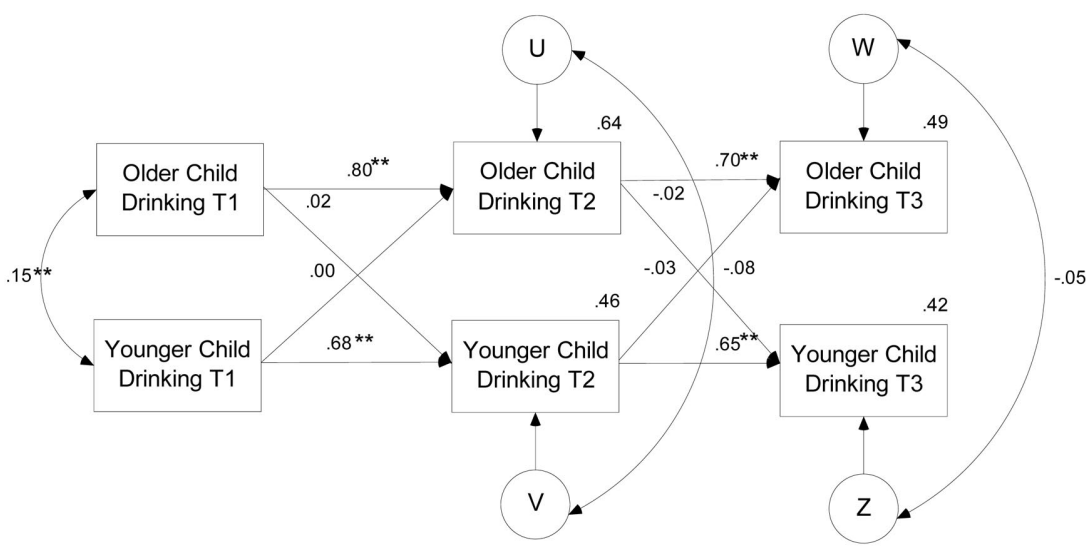

Figure 8. Friended comparison group model (standardized estimates). The friended group includes random pairs of same-grade, same-gender friended participants, neither of whom had ever nominated the other as an important peer or peer associate. $U=$ residual variance in older child's intoxication frequency at Time $2 ; V=$ residual variance in younger child's intoxication frequency at Time $2 ; W=$ residual variance in older child's intoxication frequency at Time $3 ; Z=$ residual variance in younger child's intoxication frequency at Time 3 . ${ }^{*} p<.05 .{ }^{* *} p<.01$.

influence between friends and the characteristics of partners who are particularly influential. We found that older partners were the more reliable influence agent, but we expect that future studies will identify other attributes that limit or enhance the ability and susceptibility to influence.

Interest in processes underlying homophily is more than academic. Across the adolescent years, friendships become an increasingly important feature of the child's social world. The influence of friends is matched only by that of parents, and there are several domains in which friends are more influential than parents, particularly when it comes to daily activities (Collins \& Laursen, 2004). Many delinquent and antisocial behaviors, for instance, are perpetuated in the presence of friends (Cairns \& Cairns, 1994). Identifying the relative importance of selection and socialization effects has important implications for intervention efforts, because one suggests a focus on preventing the establishment of antisocial relationships, whereas the other suggests a focus on reforming or disrupting those that have already formed. A failure to understand the origins of homophily has undermined many intervention efforts (Dishion, McCord, \& Poulin, 1999).

The comparison groups provided an estimate of age-related changes in the degree to which the entire population became more similar on problem drinking behavior. Moffitt (1993) argued that many adolescents go through a period of normative deviant behavior, where a large number of previously rule-abiding youth experiment with norm breaking. This could artificially inflate estimates of friendship homophily if changes at the level of the population are conflated with changes at the level of the dyad.

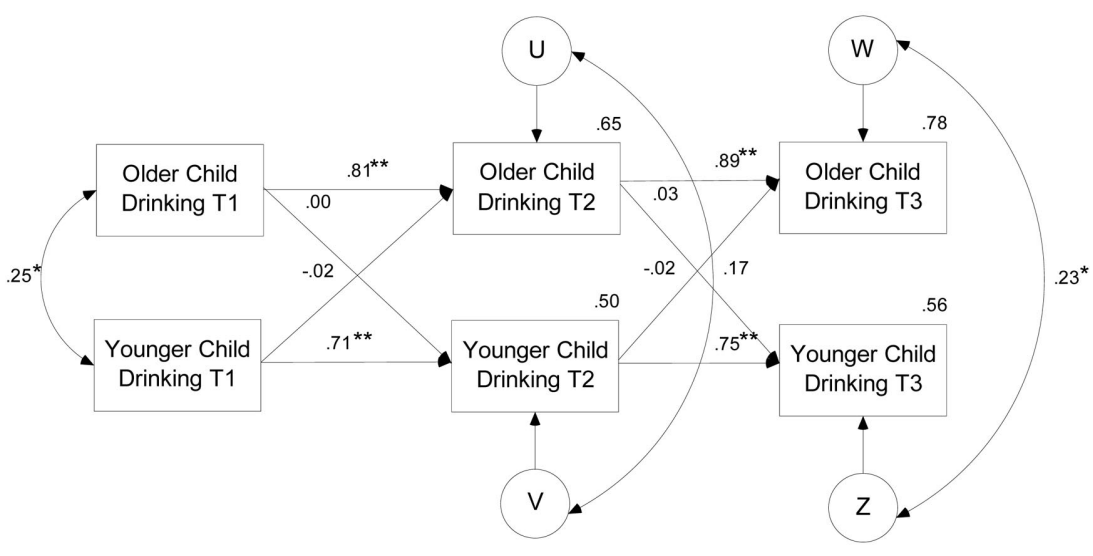

Figure 9. Friendless comparison group model (standardized estimates). The friendless group includes random pairs of same-grade, same-gender friendless participants, neither of whom had ever nominated the other as an important peer or peer associate. $U=$ residual variance in older child's intoxication frequency at Time $2 ; V=$ residual variance in younger child's intoxication frequency at Time $2 ; W=$ residual variance in older child's intoxication frequency at Time $3 ; Z=$ residual variance in younger child's intoxication frequency at Time 3 . ${ }^{*} p<.05$. ${ }^{* *} p<.01$. 


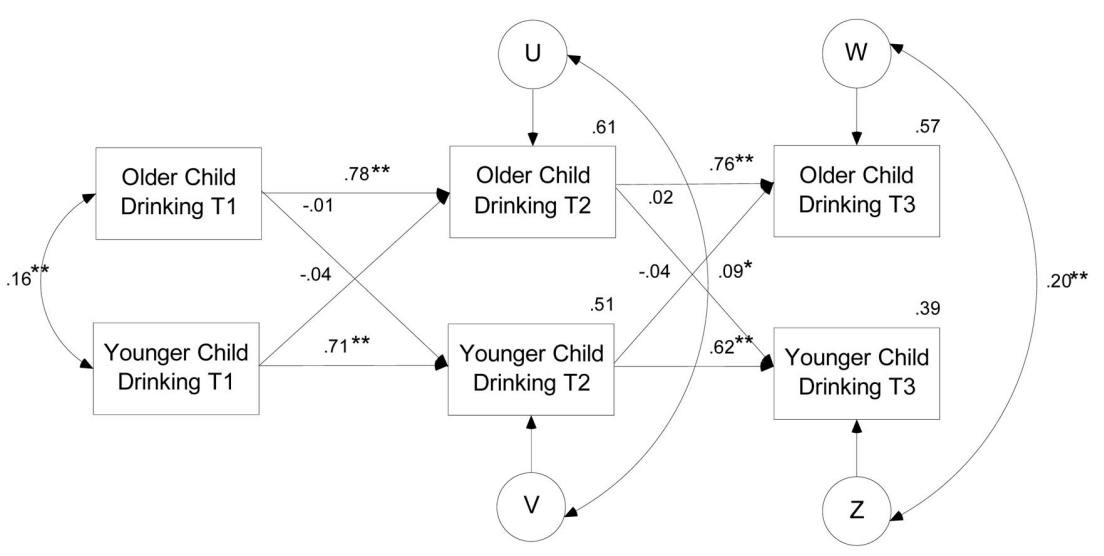

Figure 10. Total sample comparison group model (standardized estimates). The total sample group includes random pairs of same-grade, same-gender participants, neither of whom had ever nominated the other as an important peer or peer associate. $U=$ residual variance in older child's intoxication frequency at Time $2 ; V=$ residual variance in younger child's intoxication frequency at Time $2 ; W=$ residual variance in older child's intoxication frequency at Time $3 ; Z=$ residual variance in younger child's intoxication frequency at Time 3 . ${ }^{*} p<.05 .{ }^{* *} p<.01$.

Indeed, recent studies have indicated that population and peer group level changes are responsible for a small proportion of socialization effects that are sometimes attributed to friends (Jaccard et al., 2005; Steglich et al., 2006). We were unable to separate peer group level variance from dyad level variance because the multiple-model procedure does not permit individuals to participate in more than one dyad. Thus, we cannot with any certainty make claims about the relative contributions that each makes to intoxication homophily. Estimates of friendship homophily may be inflated by effects that are more appropriately ascribed to group influence processes. It is worth noting, however, that concurrent similarity effects between randomly paired same-grade, samegender youth were substantially smaller than those between friends, suggesting that population level changes account for only a small fraction of what is typically defined as friendship homophily.

Table 2

Alcohol Intoxication Frequency: Within-Dyad Correlations

\begin{tabular}{llllr}
\hline \multicolumn{1}{c}{ Friendship group } & Time $1 r$ & Time $2 r$ & Time $3 r$ & $\begin{array}{r}n \text { of } \\
\text { dyads }\end{array}$ \\
\hline Stable friends & & & & \\
Enduring (Time 1, 2, \& 3) & $.74^{* * *}$ & $.73^{* *}$ & $.70^{* *}$ & 119 \\
Nascent (Time 2 \& 3) & $.54^{* *}$ & $.68^{* *}$ & $.75^{* *}$ & 57 \\
$\quad$ Waning (Time 1 \& 2) & $.49^{* * *}$ & $.53^{* *}$ & $.41^{* *}$ & 55 \\
Unstable friends & & & & \\
Time 1 only & $.46^{* * *}$ & $.35^{* *}$ & $.42^{* *}$ & 70 \\
Time 2 only & $.68^{* *}$ & $.59^{* *}$ & $.46^{* *}$ & 62 \\
Time 3 only & $.42^{* * *}$ & $.53^{* *}$ & $.46^{* *}$ & 76 \\
Comparison & & & & \\
Friended & $.15^{* * *}$ & .07 & -.03 & 439 \\
Friendless & $.25^{* * *}$ & $.20^{*}$ & $.21^{*}$ & 106 \\
Total sample & $.16^{* *}$ & $.09^{*}$ & $.14^{* *}$ & 545 \\
\hline
\end{tabular}

Note. Comparison groups include random pairs of same-grade, samegender participants, neither of whom had ever nominated the other as an important peer or peer associate.

${ }^{*} p<.05 .{ }^{* * *} p<.01$.
The present study is not without limitations. Participants were drawn from a small community in central Sweden. Although they were representative of the population from which they were drawn, it will be up to future scholars to determine the extent to which the findings from this sample generalize to youth living in other settings, particularly those in communities that are more urban and transient. Friendship nominations and similarity estimates were obtained at annual intervals. As a consequence, estimates of selection and socialization lack precision because of the considerable variation within groups in the time between assessments and the onset of the friendship. Friendship groups were conflated with characteristics of participants. Youths who change friends with some regularity undoubtedly differ from those who keep the same friends on several personality and behavioral dimensions that are associated with intoxication frequency. Gender may moderate patterns of association. Boys were overrepresented among individuals who changed friends, and girls were overrepresented among those who kept the same friends, suggesting that results may better describe the former than the latter. Sample size limitations prevented the examination of gender as a moderator using the current sample; however, the method presented here could be extended to include gender as a moderator given a large sample size. Attrition analyses indicated that heavier drinkers were underrepresented in our sample, and indeed, a substantial proportion of the participants had never been intoxicated during the first wave of data collection. These two factors limited the variance in our model, suggesting that our findings may offer a conservative estimate of the extent to which friends share and shape drinking behavior. Finally, the current model groups friendship dyads on the basis of when they formed and dissolved. Future research might model the formation and dissolution of friendships as endogenous to peer influence.

Incorporating interdependent data into the design and analysis of longitudinal research is one of the greatest challenges facing contemporary developmental scholars. Nowhere is this challenge more pressing than in the developmental study of close relationships, which requires prospective data concerning both participants 
in a relationship. Unfortunately, interdependent data are often underutilized because of an apparent lack of appropriate analytic tools. This article describes a new application of the actor-partner interdependence model that permits interdependent data to be parsed into variance that describes over-time similarity, stability, and influence. We modified the technique to describe homophily in adolescent friendships, but it is well suited for use with many different developmental phenomena.

\section{References}

Adams, R. E., Bukowski, W. M., \& Bagwell, C. (2005). Stability of aggression during early adolescence as moderated by reciprocated friendship status and friend's aggression. International Journal of Behavioral Development, 29, 139-145.

Arbuckle, J. L. (2006). Amos (Version 7.0) [Computer program]. Chicago: SPSS.

Berndt, T. J., \& Keefe, K. (1995). Friends' influence on adolescents' adjustment to school. Child Development, 66, 1312-1329.

Cairns, R. B., \& Cairns, B. D. (1994). Lifelines and risks: Pathways of youth in our time. New York: Cambridge University Press.

Card, N. A., Little, T. D., \& Selig, J. P. (Eds.). (in press). Modeling dyadic and interdependent data in developmental research. Mahwah, NJ: Erlbaum.

Cillessen, A. H. N., Jian, X. L., West, T. V., \& Laszkowski, D. K. (2005). Predictors of dyadic friendship quality in adolescence. International Journal of Behavioral Development, 29, 165-172.

Collins, B., \& Laursen, W. A. (2004). Interpersonal conflict during adolescence. Psychological Bulletin, 115, 197-209.

Cook, W. L., \& Kenny, D. A. (2005). The actor-partner interdependence model: A model of bidirectional effects in developmental studies. International Journal of Behavioral Development, 29, 101-109.

Dishion, T. J., McCord, J., \& Poulin, F. (1999). When interventions harm: Peer groups and problem behavior. American Psychologist, 54, 1-10.

Heckman, J. J. (1979). Sample selection bias as a specification error. Econometrica, 47, 153-161.

Jaccard, J., Blanton, H., \& Dodge, T. (2005). Peer influences on risky behavior: An analysis of the effects of a close friend. Developmental Psychology, 41, 135-147.

Kandel, D. B. (1978). Homophily, selection, and socialization in adolescent friendships. American Journal of Sociology, 84, 427-436.

Kashy, D. A., \& Kenny, D. A. (2000). The analysis of data from dyads and groups. In H. T. Reis \& C. M. Judd (Eds.), Handbook of research methods in social and personality psychology (pp. 451-477). New York: Cambridge University Press.
Kenny, D. A. (1995). The effect of nonindependence on significance testing in dyadic research. Personal Relationships, 2, 65-75.

Kenny, D. A., \& Cook, W. (1999). Partner effects in relationship research: Conceptual issues, analytic difficulties, and illustrations. Personal Relationships, 6, 433-448.

Kenny, D. A., Kashy, D. A., \& Cook, W. (2006). Dyadic data analysis. New York: Guilford Press.

Kiesner, J., Kerr, M., \& Stattin, H. (2004). "Very important persons" in adolescence: Going beyond in-school, single friendships in the study of peer homophily. Journal of Adolescence, 27, 545-560.

Kline, R. B. (2005). Principles and practice of structural equation modeling (2nd ed.). New York: Guilford Press.

Laursen, B. (2005). Dyadic and group perspectives on close relationships. International Journal of Behavioral Development, 29(2), 97-100.

Laursen, B., Popp, D., Burke, W. J., Kerr, M., \& Stattin, H. (in press). Incorporating interdependence into developmental research: Examples from the study of homophily and homogeneity. In N. A. Card, T. D. Little, \& J. P. Selig (Eds.), Modeling dyadic and interdependent data in developmental research. Mahwah, $\mathrm{NJ}$ : Erlbaum.

Lazarsfeld, P. F., \& Merton, R. K. (1954). Friendship as a social process. In M. Berger, T. Abel, \& C. H. Page (Eds.), Freedom and control in modern society (pp. 18-66). Princeton, NJ: Van Nostrand.

McKnight, P. E., McKnight, K. M., Sidani, S., \& Figueredo, A. (2007). Missing data: A gentle introduction. New York: Guilford Press.

Moffitt, T. E. (1993). Adolescence-limited and life-course-persistent antisocial behavior: A developmental taxonomy. Psychological Review 100, 674-701.

Neyer, F. J. (2002). The dyadic interdependence of attachment security and dependency: A conceptual replication across older twin pairs and younger couples. Journal of Social and Personal Relationships, 19, 483-503.

Olsen, J. A., \& Kenny, D. A. (2006). Structural equation modeling with interchangeable dyads. Psychological Methods, 11, 1-15.

Persson, A., Kerr, M., \& Stattin, H. (2007). Staying in or moving away from structured activities: Explanations involving parents and peers. Developmental Psychology, 43, 97-107.

Steglich, C. E. G., Snijders, T. A. B., \& West, P. (2006). Applying SIENA: An illustrative analysis of the coevolution of adolescents' friendship networks, taste in music, and alcohol consumption. Methodology, 2, $48-56$.

Urberg, K. A., Değirmencioğlu, S. M., \& Pilgrim, C. (1997). Close friend and group influence on adolescent cigarette smoking and alcohol use. Developmental Psychology, 33, 834-844.

Received December 1, 2006

Revision received December 17, 2007

Accepted January 24, 2008 